Thr ee- di mensi onal parti $\mathrm{cl}$ e si mul at $\mathrm{i}$ on on structure for mat i on and pl asma i nst abi l i ti es in coll i si onl ess dri ven reconnection

\begin{tabular}{|l|l|}
\hline $\begin{array}{l}\text { j our nal or } \\
\text { publ i cat i on titl e }\end{array}$ & Journal of Pl asma Physi cs \\
\hline vol une & Vol . 72 \\
\hline number & I ssue 6 \\
\hline page r ange & pp. 929-933 \\
\hline year & 2006-12-01 \\
\hline URL & ht t p: //hdl . handl e. net /10655/2288 \\
\hline
\end{tabular}




\title{
Three-dimensional particle simulation on structure formation and plasma instabilities in collisionless driven reconnection
}

\author{
H. OH T A N I I,2, R. H O R I U CH I ${ }^{1,2}$ and A. I S H I Z A W A ${ }^{1}$ \\ ${ }^{1}$ Theory and Computer Simulation Center, National Institute for Fusion Science, \\ 322-6 Oroshi-cho, Toki 509-5292, Japan \\ ${ }^{2}$ The Graduate University for Advanced Studies (Soken-dai), \\ 322-6 Oroshi-cho, Toki 509-5292, Japan
}

(Received 29 August 2005 and accepted 1 March 2006)

\begin{abstract}
Structure formation and plasma instabilities in collisionless driven reconnection are investigated by three-dimensional electromagnetic (EM) particle simulation in an open system. In the early period, the lower-hybrid drift instability is excited in the periphery of the current layer. In the intermediate period, magnetic islands are created in the downstream due to magnetic reconnection, and they become unstable against a kink instability. In the late period, after the magnetic islands go out through the boundary, a wide, thin current sheet is generated and a low-frequency EM mode is excited in the central region. This mode has a frequency comparable to the ion gyration frequency, and thus it is considered to be the drift kink instability.
\end{abstract}

\section{Introduction}

Magnetic reconnection plays an important role in high-temperature plasmas, for instance, Solar corona, magnetospheric substorm and high-temperature tokamak discharge [1]. It leads to fast energy release from magnetic field to plasmas and the change of magnetic field topology. However, the mechanism of the fast energy release is not fully understood. Several computer simulations have been performed to study this mechanism. Two-dimensional particle simulation has been carried out in an open boundary model to study the triggering mechanism of collisionless reconnection from the standpoint of particle kinetic effects $[2,3]$. A variety of plasma instabilities have so far been studied as candidates for an anomalous resistivity in a current sheet [4-8]. To clarify the relationship between particle kinetic effects and anomalous resistivity due to plasma instabilities, we have developed a three-dimensional particle simulation code for magnetic reconnection in an open system 'PASMO' $[4,5]$, in which a distributed parallel algorithm is integrated for a distributed memory and multi-processor vector computer system [9].

\section{Simulation model and code}

The simulation code 'PASMO' relies on an explicit electromagnetic (EM) particlein-cell method. The initial condition is given by a one-dimensional Harris-type 
equilibrium, in which the magnetic field is parallel to $x$-axis and a function of $y$-coordinate. The distribution of particles is a shifted Maxwellian with a uniform temperature and a uniform average velocity equal to the diamagnetic drift velocity. At the upstream boundary $\left(y= \pm y_{\mathrm{b}}\right)$, ions and electrons are frozen into magnetic field, and thus the plasma inflow is driven by $\mathbf{E} \times \mathbf{B}$ drift due to a driving electric field $E_{z 0}$ applied in the $z$ direction. The boundary conditions for remaining field quantities at upstream are as follows: $E_{x}=0$ and $\partial_{y} E_{y}=0$. At the downstream boundary $\left(x= \pm x_{\mathrm{b}}\right)$, the field quantities $E_{x}, \partial_{x} E_{y}$ and $\partial_{x} E_{z}$ are continuous. Under the free boundary condition at the downstream, particles go out of and come into the system through it. The information on outgoing particles is obtained directly by observing the motion of particles at the boundary. However, we must define the number of particles which should go into the system, and the positions and velocities of these incoming particles. For these problems, we assume that the net numbers for electrons and ions are the same, and that the physical state outside is the same as that near the downstream boundary [2].

We improve the simulation code using High Performance Fortran (HPF) for a distributed memory and multi-processor vector computer system. For data layout, all field data are duplicated on each parallel process, but particle data are distributed among them. An operation for outgoing and incoming particles is performed in each processor, and the only reduction operation for the number of particles is executed in data transfer. This adequate treatment reduces the amount and frequency of data transfer. The compiler-directive line listvec in the gather process dramatically decreases the memory size and improves the vector operation in an NEC SX system (vector operation ratio is about $99.5 \%$ and vector length turns 240 and over) [9].

\section{Simulation results}

In this section, we show simulation results of collisionless driven reconnection. Simulation parameters are as follows. The total number of particles is 72 million at initial stage, the simulation box size is $252 \lambda_{\mathrm{D}} \times 63 \lambda_{\mathrm{D}} \times 192 \lambda_{\mathrm{D}}$, the scale length of current layer $L$ is $25.2 \lambda_{\mathrm{D}}$ at the initial time, the thermal velocities of electron and ion are respectively $0.25 c$ and $0.025 c$, where $c$ is the speed of light. $\lambda_{\mathrm{D}}$ is Debye length. The mass ratio $m_{\mathrm{i}} / m_{\mathrm{e}}$ is 100 and the driving field $E_{z 0}$ is $-0.04 B_{0}$, where $B_{0}$ is a constant.

Figures 1 and 2 show the isosurface of $B_{x}^{2}+B_{y}^{2}$ and streamline of magnetic field, and the isosurface of current density and wave corresponding to Fourier mode of the electric field $E_{z}^{n=7}$ at $t \omega_{\text {ce }}=181.25$, respectively. Figures 3 and 4 show the same figures as Fig. 1 but at $t \omega_{\text {ce }}=331.25$ and 1312.50 , respectively.

As the simulation proceeds, the driving field imposed at the upstream boundary penetrates into the current sheet. In the relatively early period $\left(t \omega_{\mathrm{ce}}=181.25\right)$, a wavy structure appears in the periphery of current layer (Figures 1 and 2). Based on the spectrum analysis of $E_{z}$ in the periphery of the current layer, the dominant mode of the wave is $n=7$, and the peak frequency is consistent with the lower hybrid frequency $\left(\omega_{\mathrm{LH}}\right)$. Thus, this mode is identified as the lower hybrid drift (LHD) mode [6,7]. In this way, the driving field infiltrates into the current sheet owing to particle kinetic effects, and triggers magnetic reconnection when it reaches the center. At that time, the current density in the central region becomes peaked (Fig. 2). In the middle period $\left(t \omega_{\mathrm{ce}}=331.25\right)$, magnetic islands are created 


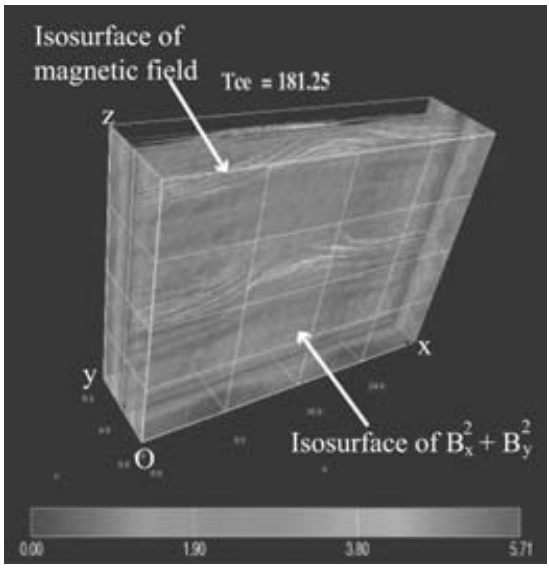

Figure 1. Isosurface of $B_{x}^{2}+B_{y}^{2}$ and streamline of magnetic field at $t \omega_{\mathrm{ce}}=181.25$.

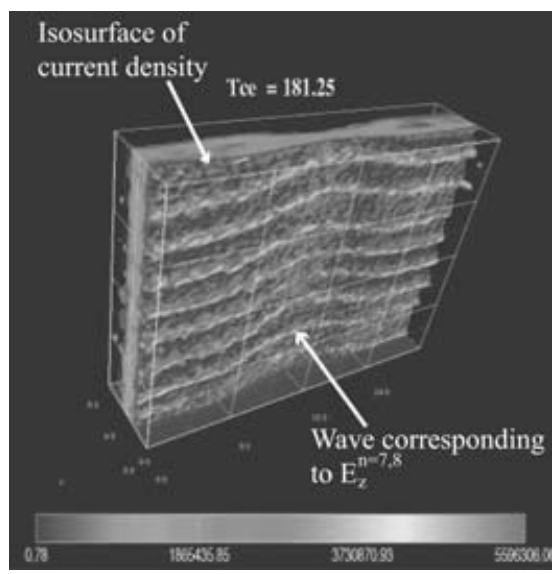

Figure 2. Isosurface of current density and wave corresponding to $E_{z}^{n=7}$ at $t \omega_{\mathrm{ce}}=181.25$.

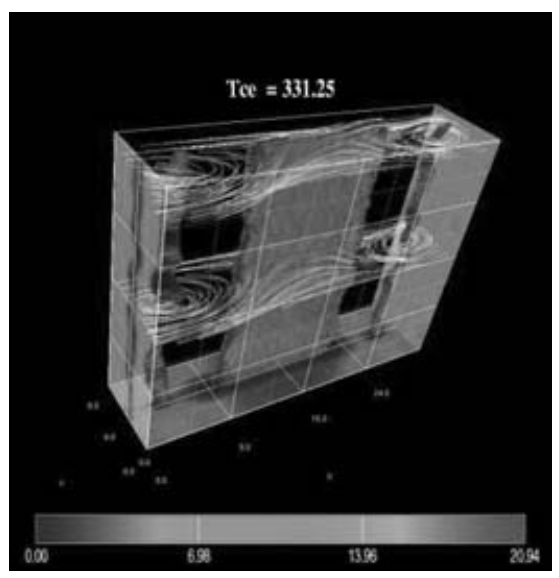

Figure 3. The same as Fig. 1 but at $t \omega_{\mathrm{ce}}=331.25$. 


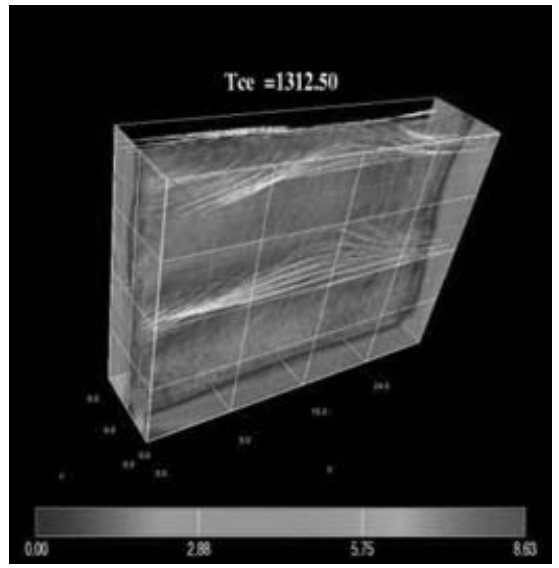

Figure 4. The same as Fig. 1 but at $t \omega_{\mathrm{ce}}=1312.50$.

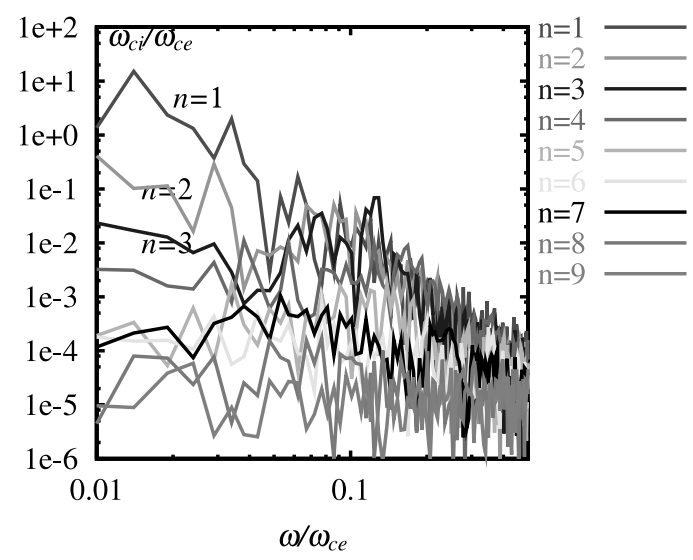

Figure 5. Spectrum of Fourier mode of $B_{x}$ at the center region.

in the downstream as a result of magnetic reconnection. While they go towards the downstream boundary, they become unstable against an ideal kink instability (Fig. 3). After they move out through the downstream boundary, a wide, thin current sheet is generated, and then a low-frequency EM instability is excited near the central region (Fig. 4). The wave number of the dominant mode is $n=1$ and its frequency is comparable to the ion gyration frequency (Fig. 5). These results are in good agreement with the linear analysis of the drift kink (DK) mode [8]. Figure 6 shows the maximum growth rate $(\gamma)$ of DK mode as a function of $m_{\mathrm{i}} / m_{\mathrm{e}}$, where an open circle corresponds to the simulation result and the linear analysis of the DK mode is plotted as solid curve for comparison. The growth rate from the simulation is larger than that of the linear analysis. The reason for the disagreement and the simulation growth rate between the linear theory may be due to the fact that the current sheet evolves dynamically with time. One of the important issues of the DK mode is its dependence on the mass ratio $m_{\mathrm{i}} / m_{\mathrm{e}}$. This problem will be studied in the near future. 


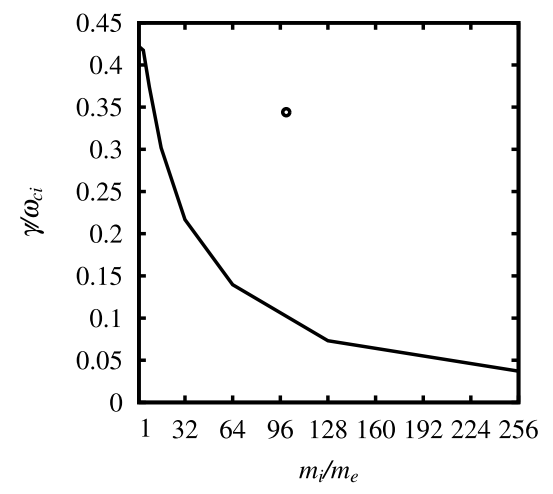

Figure 6. Growth rate of DK mode as a function of $m_{\mathrm{i}} / m_{\mathrm{e}}$. An open circle is the simulation result and solid curve is by the linear analysis [8].

\section{Conclusions}

To investigate the behavior of magnetic reconnection, we developed a three-dimensional full EM particle simulation code in an open boundary system "PASMO". Moreover, we improved it using High Performance Fortran (HPF) for a distributed memory and multi-processor vector computer system, and succeed in opening a path for a large-scale simulation. From the simulation results, it is found that in the early period the LHD mode is excited in the periphery of current layer, but the anomalous resistivity is not generated by the LHD mode. After the current sheet is modified by this LHD mode, a DK instability is excited near the central region in the late period and sustained for a long time. It is also found that the DK mode plays an important role in generating the anomalous resistivity in this current sheet.

\section{References}

[1] Biskamp, D. 2000 Magnetic Reconnection in Plasmas. Cambridge: Cambridge University Press.

[2] Pei, W., Horiuchi, R. and Sato, T. 2001 Long time scale evolution of collisionless driven reconnection in a two-dimensional open system. Phys. Plasmas 8, 3251-3257.

[3] Ishizawa, A., Horiuchi, R. and Ohtani, H. 2004 Two-scale structure of the current layer controlled by meandering motion during steady-state collisionless driven reconnection. Phys. Plasmas 11, 3579-3585.

[4] Horiuchi, H. and Sato, T. 1999 Three-dimensional particle simulation of plasma instabilities and collisionless reconnection in a current sheet. Phys. Plasmas 6, 45654574.

[5] Horiuchi, R., Ishizawa, A. and Ohtani, H. 2004 Dynamical evolution of thin current sheets in a three-dimensional open system. J. Plasma Fusion Res. SERIES 6, 614-617.

[6] Huba, J. D., Gladd, N. T. and Papadopulos, K. 1978 Lower-hybrid-drift wave turbulence in the distant magnetotail. J. Geophys. Res. 83, 5217-5226.

[7] Ozaki, M., Sato, T., Horiuchi, R. and Group, C. S. 1996 Electromagnetic instability and anomalous resistivity in a magnetic neutral sheet. Phys. Plasmas 3, 2265-2274.

[8] Daughton, W. 1999 The unstable eigenmodes of a neutral sheet. Phys. Plasmas 6, 13291343.

[9] Ohtani, H., Ishiguro, S., Horiuchi, R., Hayashi, Y. and Horiuchi, N. 2005 In: Development of Electromagnetic Particle Simulation Code in an Open System. Lecture Notes Comput. Sci. Berlin: Springer, in press. 